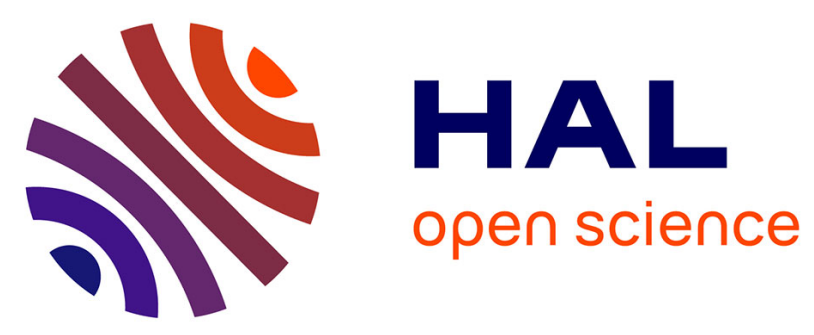

\title{
On optimal polling policies
}

Zhen Liu, Philippe Nain, Don Towsley

\section{To cite this version:}

Zhen Liu, Philippe Nain, Don Towsley. On optimal polling policies. [Research Report] RR-1541, INRIA. 1991. inria-00075021

\section{HAL Id: inria-00075021 https://hal.inria.fr/inria-00075021}

Submitted on 24 May 2006

HAL is a multi-disciplinary open access archive for the deposit and dissemination of scientific research documents, whether they are published or not. The documents may come from teaching and research institutions in France or abroad, or from public or private research centers.
L'archive ouverte pluridisciplinaire HAL, est destinée au dépôt et à la diffusion de documents scientifiques de niveau recherche, publiés ou non, émanant des établissements d'enseignement et de recherche français ou étrangers, des laboratoires publics ou privés. 


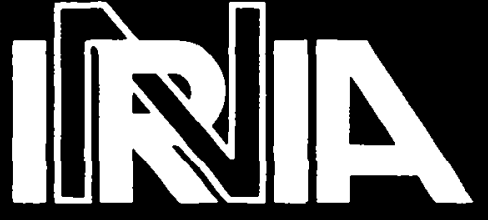

UNITÉ DE RECHERCHE INRIA-SOPHIA ANTIPOLIS

\title{
Rapports de Recherche
}

\author{
$N^{\circ} 1541$
}

Programme 1

Architectures parallèles, Bases de données, Réseaux et Systèmes distribués

\section{ON OPTIMAL POLLING POLICIES}

Institut National de Recherche en Informatique et en Automatique

Domaine de Voluceau

Rocquencourt

B.P.105

78153 Le Chesnay Cedex

France

Tél.:(1) 39635511
Zhen LIU

Philippe NAIN

Don TOWSLEY

Octobre 1991 


\title{
POLITIQUES DE "POLLING" OPTIMALES
}

\author{
Zhen LIU ${ }^{1}$, Philippe NAIN', Don TOWSILI:Y3 \\ ${ }^{1,2}$ INRIA, Centre de Sophia Antipolis \\ 06565 Valbonne Cedex, France \\ ${ }^{3}$ Department of Computer and Information Science \\ University of Massachusetts. Amherst, MA 0100:3, USA
}

\begin{abstract}
Résumé
Dans un système de files d'attente monoserveur de type polling le servour visite les files suivant une politique de routage. Le comportement du serveur dans chacpue file d'attente visitée est spécifié par la politique de service. En particulier, la politique de service indique si le serveur doit servir ou non des clients et, dans le premier cas, le nombre de clicnts qu'il doit servir. Une politique d'ordonnancement est la donnée d'une politique de routage et d'une politique de service. L'objectif de cet article est de trouver des politiques d'ordonnancement qui minimisent, pour l'ordre stochastique, la charge totale du système et le nombre total de clients en attente de traitement. Ce problème d'optimisation est décomposé en trois sous-problèmes: déterminer le comportement optimal (servir, changer de file, rester inactif) du serveur lorsqu'il visite une file non vide; déterminer le comportement optimal du serveur (changer de file, rester inactif) lorqu'il visite une file vide; déterminer le routage optimal (choix de la prochaine file à visiter) lorsque le serveur vient de vider une file et qu'il décide de changer de file. Sous des hypothèses très générales, nous montrons que les politiques d'ordonnancement optimales pour le premier sous-problème sont nécessairement gloutonnes et exhaustives, ce qui signifie que le scrveur doit servir les clients de chaque file sans discontinuer, jusqu'au dernier. Dans le cas d'un système symétrique, nous montrons que les politiques d'ordonnancement optimales pour le second sous-problème sont des politiques patientes, dans le sens ou le serveur doit rester dans la dernière file visitée lorsque le système est vide. Si le système est discrétisé nous montrons en outre que les politiques conservatrices (le servenr est tonjours actif lorsque le système est non vide) et impatientes (le serveur quitte une file dès qu'elle est vide) sont optimales. Enfin, si le système est symétrique nous prouvons que les politiques de routage qui optimisent le troisième sous-problème sont celles qui envoient le serveur vers la file la plus longue pour l'ordre stochastique.
\end{abstract}

Mots-Clés: Polling; Files d'attente multidimensionnelle; Ordonnancement stochastique; Ordre stochastique; Couplage. 


\title{
ON OPTIMAL POLLING POLICIES*
}

\author{
Zlien LIU ${ }^{1}$, Philippe NAIN², Don 'TOWSLFY'3 \\ ${ }^{1,2}$ INRIA, Centre de Sophia Antipolis \\ 0656.5 Valbonne Cedex, France \\ ${ }^{3}$ Department of Computer and Information Science \\ University of Massachusetts, Amherst, MA 01003, USA
}

\begin{abstract}
In a single server polling system, the server visits the queues according to a routing policy and while at a queue serves some or all of the customers there according to a service policy. A polling (or scheduling) policy is a sequence of decisions on whether to serve a customer, idle the server, or switch the server to another quete. The goal of this paper is to find polling policies that stochastically minimize the unfinished work and the number of customers in the system at all time. This optimization problem is decomposed into three subproblems: determine the optimal action (i.e., serve, switch, idle) when the server is at a nonempty queue; determine the optimal action (i.e., switch, idle) when the server empties a queue; determine the optimal routing (i.e., choice of the queue) when the server empties a queue and decides to switch.

Under fairly general assumptions, we show for the first subproblem that optimal policies are greedy and exhaustive, i.e., the server should neither idle nor switch when it is at a nonempty queue. For the second subproblem, we prove that in symmetric polling systems patient policies are optimal, i.e., the server should stay idling at the last visited queue whenever the system is empty. When the system is slotted, we further prove that non-idling and impatient policies are optimal. For the third subproblem, we establish that in symmetric polling systems optimal policies belong to the class of Stochastically Largest. Queue (SLQ) policies. A SLQ policy is one that never routes the server to a queue known to have a queue length that is stochastically smaller than that of another queuc. This result implies, in particular, that the policy that routes the server to the queue with the largest queue length is optimal when all queue lengths are known and that the cyclic routing policy is optimal in the case that the only information available is the previous decisions.
\end{abstract}

\footnotetext{
*This work was supported in part. by NSF under contract ASC-8802764
} 


\section{Introduction}

In this paper, we consider the problem of scheduling a server in a polling system. We model this system as $N$ quenes attended by a single server. The server visits the quenes according to some rule. While at a quene, the server serves some or all of the customers again according to some rule. A polling (or scheduling) policy consists of a scries of decisions on whether to serve a customer, to idle the server, or to switch to another (jueuc. 'These decisions are made based on partial knowledge of the state of the quene (i.e., queue occupancies, past arrival patterns) and on past scheduling decisions.

Polling systems are frequently used to model computer and communication networks (cf. Takagi [7]). The performance analysis of these systems has been the subject of a large number of papers (more than 450 references are included in 'lakagi's survey paper [8]). These analyses have focussed on a large number of routing policies including cyclic and probabilistic routing and on a large number of service policies such as exhaustive, gated and limited service.

However, very few papers have focused on the problem of developing optimal scheduling policics, except for the simple $u c$ rule which minimizes some discounted cost function when there is no switch-over time. One of the first attempts to optimize polling systems with switch-over times was made by Hofri and Ross [4]. They show for a two queue model that the policy that minimizes the sum of discounted switch-over times and the holding cost is exhaustive service in a nonempty queue and of threshold type for switching from an empty queue to another. Browne and Yechiali [3] provide a semi-dynamic policy where the server chooses a visiting order of the queucs at the beginning of each cycle in order to minimize the cycle time. A similar approach is presented by Browne and Yechiali [2] for queues with unit buffer and losses of customers. They show that an index rule policy minimizes the sum of holding costs and customer loss costs over a cycle. In Levy et al. [5], it is proved that the exhaustive policy dominates all other service policics, under the assumptions that the server does not wait idling at a queue and that the server switches to the next queue once a queue is emptied. In [6] Liu and Nain consider a particular polling system arising from the videotex system. Depending on the amount of information available to the controller, they identify optimal scheduling policies under fairly general assumptions in the case when there are no switch-over times. Last, in [9], Towsley, et al. prove that the policy that serves the quene with the largest queue length minimizes the number of customers that are lost when queues have finite and equal buffer cpacities, arrivals to each quene are governed by a class of statistically identical processes, and switching times are negligible.

In this paper, we study the case where the switch-over times are strictly positive. The aim is to find polling policies that stochastically minimize the unfinished work and the number of customers in the system at all time. This optimization problem reduces to answering the following questions:

(1) when the server is at a nonempty queue, should it serve a customer of that queue or not?

(2) when the server empties a queve, should it switch or not? 
(3) when the server empties a quene and decides to switch to another queue, which queve should it switch to?

The paper is organized as follows. Notation and assumptions are introduced in Section 2. The answer to question (1) is given in Sections 3 and 4 . We show, under failly general assumptions, that optimal policies are greedy and exhaustive. In other words, when the server is at a nonempty quene, it should neither idlo nor switch until this quene is empty.

Section 5 answers question (3) in the case where the polling system is symmetric. We establish that the optimal policy is a Stochastically Larycst Queue (SLQ) policy. Here a SLQ policy is the one that never visits a queue known to have a quene length that is slochastically smaller than that of another queue. From this result, we obtain that the routing policy that moves the server to the queue with the largest queue length is optimal when all queue lengths are known at all times. In the case that the only available information is the previous decisions, we show that the cyclic routing policy is optimal. We also consider other applications where the quene length information is delayed.

Last, in Sections 6 and $T$ we address question (2). Wo prove that in symmetric polling system patient policies are optimal, i.e., the server always stays idling at the last visiled queue whenever the system is empty. If in addition the svistem is slotted and that the switch-over times are one time unit, then non-idling as well as impatient policies are shown to be optimal, i.e., the server always switches whenever it empties a queue.

\section{Notation and Assumptions}

All of the random variables (r.v.'s) considered in this paper are defined on a fixed probability triple $(\Omega, \mathcal{F}, P)$. Let $\mathbb{I N}$ and $\mathbb{I N}_{+}$be the set of nomnegative integer numbers and strictly positive integer numbers, respectively. Define $\mathrm{IR}:=(-\infty,+\infty), \mathrm{IR}_{+}:=[0,+\infty)$ and $\mathrm{I}:=\{1,2, \ldots, N\}$.

Input sequences. For $n \geq 1, i \in \mathbb{I}$, let

- $a_{n}$ be the arrival time of the $n$-th customer, $0 \leq a_{1}<a_{2}<\cdots$;

- $u_{n}$ be the index of the queue at which the $n$-th customer arrives;

- $\sigma_{n}^{i}>0$ be the amount of service required by the $n$-th arriving customer at queue $i$. We shall assume throughout this paper that $\left\{\sigma_{n}^{i}\right\}_{n=1}^{\infty}$ is a sequence of independent and identically distributed (i.i.d.) r.v.'s for all $i \in$ I. Further, $\left\{\sigma_{n}^{1}\right\}_{n=1}^{\infty}, \ldots,\left\{\sigma_{n}^{N}\right\}_{n=1}^{\infty}$ are assumed to be mutually independent sequences;

- $\theta_{n}^{i, j}>0$ be the duration of the $n$-th switching period between queue $i$ and quene $j$ for all $j \in \mathbf{I}, i \neq j$. 
In the secuel, the notation A1, A2, A3. A4 and A5 will be used to denote the following assumptions:

A1 The sequences of r.v.'s $\left\{a_{n}, u_{n}\right\}_{n=1}^{\infty},\left\{\sigma_{n}^{i}, i \in \mathbb{I}\right\}_{n=1}^{\infty}$, and $\left\{\theta_{n}^{i, i},(i, j) \in \mathrm{I}^{2}, i \neq j\right\}_{n=1}^{\infty}$ are mutually independent;

A2 The sequences of r.v.'s $\left\{a_{n}\right\}_{n=1}^{\infty},\left\{u_{n}\right\}_{n=1}^{\infty},\left\{\sigma_{n}^{i}, i \in \mathbf{I}\right\}_{n=1}^{\infty}$, and $\left\{\theta_{n}^{i, j},(i, j) \in \mathbf{I}^{2}, i \neq j\right\}_{n=1}^{\infty}$ are mutually independent;

A3 The secuence $\left\{\sigma_{n}^{i}, i \in \mathbf{I}\right\}_{n=1}^{\infty}$ is an i.i.cl. secpuence of r.v.'s;

A4 The sequence $\left\{\theta_{n}^{i, i},(i, j) \in \mathbf{I}^{2}, i \neq j\right\}_{n=1}^{x}$ is an i.i.d. serpuence of r.v.'s;

A5 The sequence $\left\{u_{n}\right\}_{n=1}^{\infty}$ is an i.i.d. sequence of r.v.'s such that $\mathrm{P}^{\prime}\left(u_{n}=i\right)=1 / N$ for all $i \in \mathbf{I}$.

The polling system fulfilling assumptions A2, A3, A4 and A5 is called a symmetric polling system.

Service policy. At every queue the service policy can be arbitrary as long as it is nonpremptive and work conserving (i.e., no work can be crcated or destroyed). Instances of service policies are the exhaustive, gated and random service policies (see Takagi [ 7$]$ ). Because of our assumption that the service times at a given queue are i.i.d. r.v.'s, we shall not need to specify the order of service (e.g., FIFO, random).

Admissible polling policy. At any decision cpoch (see below) the polling policy must decide either to move the server to another queue and to provide the queve index to which the server is sent, or to ask the server to idle at the queue at which he resides, or to ask the server to serve a customer at the queue at which he resides (provided it is nonempty) according to the enforced service policy at that queue.

The decision epochs are the service completion epochs, the epochs where the switching periods end (or equivalently, the instants when the server arrives at a queue), and the epochs where the idle periods end.

Define $\mathcal{A}:=\{0,1,2\} \times\{1,2, \ldots, N\}$ to be the decision space.

For any polling policy $\pi$, let $\pi_{n}^{\epsilon}$ denote the $n$-th decision epoch, and let $A_{n}^{*}:=\left(\pi_{n}^{a}, \pi_{n}^{q}\right) \in \mathcal{A}$ denote the $n$-th decision, where

- $\pi_{n}^{a}=0$ (resp. $\pi_{n}^{a}=1, \pi_{n}^{a}=2$ ) if the $n$-th decision is to keep the server idle (resp. serve a customer provided the queue is nonempty, move the server to another queue). We shall assume that the first decision takes place at time 0 . i.e., $\pi_{1}^{\epsilon}=0$;

- $\pi_{n}^{q}$ is the index of the queue where the server is switching to if $\pi_{n}^{a}=2$ : if $\pi_{n}^{a} \in\{0,1\}$ then $\pi_{n}^{q}$ indicates the current location of the server (i.e., $\pi_{n}^{q}=\pi_{n-1}^{q}$ ). Without loss of generality, we assume that the server is located at queue 1 at time 0 . 
To complete the definition of a polling prolicy we need to specify how the derision epochs $\left\{\pi_{n}^{\infty}\right\}_{n=1}^{\infty}$ are chosen by the policy. Define

$$
s_{n}^{\pi}:=\sum_{m=1}^{n-1} 1\left(\pi_{m}^{n}=1\right),
$$

to be the number of service periods (or departures) in $\left[0, \pi_{n}^{c}\right)$ under policy $\pi$. Note that $s_{n}^{\pi}$ includes the customer that is served in $\left[\pi_{n-1}^{e}, \pi_{n}^{c}\right)$ il $\pi_{n-1}^{n}=1$. We also define $\sigma_{m}^{\pi}, \sigma_{m}^{\pi} \in\left\{\sigma_{n}^{i}, i \in \mathbf{I}\right\}_{n=1}^{\infty}$, to be the $m$-th service time delivered by the server under policy $\pi, m \geq 1$.

Similarly, let

$$
k_{n}^{\pi}:=1+\sum_{n=1}^{n-1} 1\left(\pi_{m}^{u}=2\right)
$$

be the number of queues visited by the server in the interval of time $\left[0, \pi_{n}^{\prime}\right], n \geq 1$. Note that $k_{n}^{\pi}$ includes the quene to which the server arrives at time $\pi_{n}^{e}$ (i.e, quene $\pi_{n-1}^{q}$ ) if $\pi_{n-1}^{n}=2$. We also define $\theta_{m}^{\pi}, \theta_{m}^{\pi} \in\left\{\theta_{n}^{i, j},(i, j) \in \mathbf{I}^{2}, i \neq j\right\}_{n=1}^{\infty}$, to be the $m$-th switch-over time duration under policy $\pi$.

With the above notation, the difference $\pi_{n+1}^{+}-\pi_{n}^{*}$ when $\pi_{n}^{a} \neq 0$ is given by

$$
\pi_{n+1}^{e}-\pi_{n}^{e}= \begin{cases}\sigma_{s_{n+1}^{\pi}}^{\bar{\pi}}, & \text { if } \pi_{n}^{u}=1 \\ \theta_{k_{n}^{\pi}}^{\pi}, & \text { if } \pi_{n}^{u}=2 .\end{cases}
$$

Relation (2.3) directly follow from the definition of the service time and switch-over time processes. Note that the case $\pi_{n}^{a}=1$ follows from the assumption that the service policy at cach queue is nonpreemptive.

In order to define the duration of an idle period as well as the notion of admissible policies, we introduce the history of a policy. For all $n \geq 1$, let

$$
H_{n}^{\pi}:=\left\{\left(A_{m}^{\pi}\right)_{m=1}^{n-1},\left(\pi_{m}^{\epsilon}\right)_{m=1}^{n} \cdot\left(Q_{j}^{\pi}\left(x_{j}\right)\right)_{, \in \mathbf{I}} ; x_{i} \leq F_{i, n}^{\pi}, i \in \mathrm{I}\right\}
$$

be the history of policy $\pi$ up to time $\pi_{n}^{\epsilon}$, where $Q_{j}^{j}(t)$ is the number of customers at queue $j$ under $\pi$ at time $t$ including the customer in service, if any, and $F_{i, n}^{\pi}$ is the last time in $\left[0, \pi_{n}^{\epsilon}\right]$ that the controller has received queue-length information on queue $i$. More precisely.

$$
F_{i, n}^{\pi}:=f_{k_{n}^{\pi}, n}^{i}\left(\left(j_{n l}^{\pi}\right)_{m=1}^{k_{n}^{\pi}},\left(\tau_{m}^{\pi}\right)_{m=1}^{k_{n}^{\pi}},\left(t_{m}^{\pi}\right)_{m=1}^{k_{i n}^{\pi}-1}, \pi_{n}^{e}\right)
$$

where

- $j_{m}^{\pi}$ is the index of the $m$-th queue that has been visited by the server, $m \geq 1$;

- $\tau_{m}^{\pi}$ is the time when the $m$-th visit of the server to a queue has started. $m \geq 1$;

- $t_{m}^{\pi}$ is the time when the $m$-th visit of the server to a queue has ended, $m \geq 1$. 
Note that $k_{n}^{\pi},\left(\tau_{m}^{\pi}\right)_{m=1}^{k_{n}^{\pi}}$ and $\left(t_{m}^{\pi}\right)_{m=1}^{k_{n}^{\pi}-1}$ (an be computed from $\left(A_{m}^{\pi}\right)_{m=1}^{n-1}$ and $\left(\pi_{m}^{\prime}\right)_{m=1}^{n}$.

In (2.5) $f_{l, n}^{i}$ is any mapping $\mathbb{I N}^{l} \times \mathbb{I R}_{+}^{2 l}-\mathbb{I R}_{+}$such that for all $i \in \mathbf{I}, n \geq 1 . l \geq 1 . \mathbf{v}=\left(v_{1}, \ldots, v_{3 l}\right) \in$ $\mathrm{N}^{l} \times \mathrm{IR}_{+}^{2 l}$ :

P1 $f_{l, n}^{i}(\mathrm{v})$ is nondecreasing as a function of $n_{m}$ for all $m=l+1, \ldots, 3 l$;

P2 $\quad f_{l, n}^{i}(\mathbf{v}) \leq f_{l, n+1}^{i}(\mathbf{v})$

P3 $h_{l}^{i}(\mathbf{v}) \leq f_{l, n}^{i}(\mathbf{v}) \leq v_{3 l}$, where

$$
h_{l}^{i}(\mathbf{v}):= \begin{cases}\max _{1 \leq k \leq 1}\left\{w_{2 l+h}: v_{k}=i\right\}, & i \in\left\{v_{1}, \cdots, v_{l}\right\} \\ 0, & \text { otherwisc }\end{cases}
$$

In terms of the history (2.4) these properties have the following interpretation: P1 and P2 imply that at each decision epoch the controller lias as least as much information as at the previous decision epoch (the controller learns more and more about the system when the time goes on). The first inequality in P3 implies that at any decision epoch the controller knows (at least) the history of each queue up to the last visit of the server to that quene. The second incyuality in P3 ensures that the policy is nonanticipative in the sense that no information on the future is available at any decision epoch. Further, P3 also implies that at any decision epoch the server knows the state of the queue that it is visiting. This follows from the fact that $f_{l, n}^{i}(v)=v_{3 l}$ when $h_{l}^{i}(v)=v_{3 l}$. In particular, this information will enable the controller not to make the decision to serve an emply queue.

The mapping $f_{l, n}^{i}$ can describe various types of information structures, including those with:

1. Complete information. $f_{l, n}^{i}(\mathbf{v})=v_{3 l}, \forall i, l, n$. In other words, the queue lengths of all of the quenes are known to the controller at all times.

2. Partinal information. $f_{l, n}^{i}(\mathbf{v})=h_{l}^{i}(\mathrm{v}), \forall i, l, n$. This corresponds to a system in which the controller knows the queue length history of each queue only up until the last time the server visited that queue.

3. Periodic information. $f_{l, n}^{i}(\mathrm{v})=\max \left\{d\left\lfloor v_{3 l} / d\right\}, h_{l}^{i}(\mathrm{v})\right\}, \forall i, l, n$. Here the controller is periodically updated regarding the queue length history of all queues at intervals of length $d$.

4. Delayed information. $f_{l, n}^{i}(\mathrm{v})=\max \left\{v_{3 l}-d, h_{l}^{i}(\mathrm{v})\right\}, \forall i, l, n$. Here the controller knows the queue length history of each queue up until $d$ time units in the past. or the last time that the queue was visited, whichever provides more information.

5. Nearest neighbor information. The queues are organized in a logical ring. Whenever the server switches to queue $i$, the controller becomes aware of the quene length history of queues 
$i-1$ and $i+1$ as well as that of $i . f_{l, n}^{i}(\mathbf{v})=\max \left\{h_{l}^{i-1}(\mathbf{v}), h_{l}^{i}(\mathbf{v}), h_{l}^{i+1}(\mathbf{v})\right\}, \forall i, l, n$, where addition and subtraction on the puene index is modulo $N$.

We now come back to the duration of an iclle period (i.e., value of $\pi_{n+1}^{c}-\pi_{n}^{c}$ when $\pi_{n}^{a}=0$ ). We shall not give a formal definition of the process that generates the idle period duration since we shall allow for rather general idle periods, including interruptible idle periods. Roughly speaking, the duration of the idle period $\left[\pi_{n,}^{c}, \pi_{n+1}^{c}\right)$ is a r.t. that may depend on the history $\left(I_{n}^{\pi}, A_{n}^{\tau}\right)$ as well as on the information that the controller may receive while the server is illing (customer arrivals, typically). However, the amount of information that the controller receives in $\left[\pi_{n}^{\prime}, \pi_{n+1}^{e}\right)$ must belong to the history $h_{n+1}^{\pi}$ (i.e., the information collected by the controller cammot be lost).

Iet $\mathrm{HH}_{n}^{\pi}$ be the sot of all histories up to time $\pi_{n}^{*}$.

We define a randomizer polling policy $\pi$ in be a sequence $\left\{\pi_{n}\right\}_{n=1}^{\infty}$ of conditional probability measures on $\mathcal{A}$ given $H_{n}^{\pi}$ satisfying the constraints $\pi_{n}\left(\mathcal{A} \mid H_{n}^{\pi}\right)=1$ for all $H_{n}^{\pi} \in I I_{n}^{\pi}, n \geq 1$. A deterministic polling policy $\pi$ is a seguence of measurable functions $\left\{\pi_{n}\right\}_{n=1}^{\infty}$ from $\| f_{n}^{\pi}$ into $\mathcal{A}$. Any polling policy that is either randomized or deterministic is called an admissible policy.

Let $\Pi$ be the set of all admissible policies. A policy $\pi \in \| l$ is said to be:

- non-idling if the server never idles when the system is nonempty. Jet $\Upsilon \subset$ Il be the set of all such policies;

- greedy if the server never idles at a nonempty queue. Let I C II be the set of all such policies;

- exhaustive if the server never leaves a nonempty quene. Let $\Xi \subset$ II be the set of all such policies;

- patient if the server stays at the last visited queue when the system is cmpty. Let $\Psi \subset$ II be the set of all such policies.

- impatient if the server leaves a gueue as soon as it is empty. Let $\Delta \subset$ II be the set of all such policies.

Performance metrics. The performance metrics to be considered in this paper are:

- $U^{\pi}(t)$, the total unfinished work in the system at time $t$ under $\pi \in I I$;

- $Q^{\pi}(t):=\sum_{i \in \mathrm{I}} Q_{i}^{\pi}(t)$, the total number of customers in the system at time $t$ under $\pi \in$ II.

We shall say in the sequel that a real-valued r.v. $X$ is stochastically smaller than a real-valued r.v. if $\mathrm{P}(X \leq x) \geq \mathrm{P}(Y \leq x)$ for all $x \in \mathbb{R}$. In that case the notation $X \leq s t Y^{\prime}$ will be used. 


\section{Optimality of Greedy Policies}

We first compare the decisions between "serving" and "idling" when the server is at a nonempty queve. Under appropriate assumptions, we show that any policy in II can be improved by a greedy policy in the sense of stochastic minimization of the unfinished work and the number of customers in the system at all time. In other words, in order to minimize these criteria, the server cannot idle at a nonempty queue.

Proposition 3.1 Assume that A1 holds. Then, for any policy $\pi \in \|$ there exists a policy $\xi \in \Gamma$ such that.

$$
\left(l^{\xi}(l) \leq_{\text {si }}\left(l^{\pi}(l)\right.\right.
$$

for all $t \geq 0$.

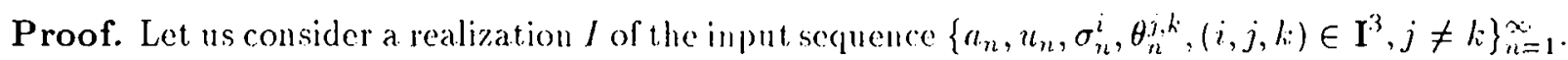
Let $\pi$ be an arbitrary policy in $\pi \in \mathrm{II}-\Gamma$ (if $\pi \in \mathrm{I}$ then take $\xi=\pi$ ) and let $\pi$ run on the input. sequence $I$.

On the sample path $\mathcal{I}$ gencrated by letting $\pi$ run on the input seguence $I$, let $n \geq 1$ be the smallest integer such that $\pi_{n}^{u}=0$ and $Q_{\pi_{n}^{u}}^{\pi}\left(\pi_{n}^{f}\right)>0$. Let $m$ be the smallest integer such that $n<m, \pi_{m}^{u}=1$, and $\pi_{m}^{q}=\pi_{n}^{q}$. Note that if $m=\infty$ then the server does not server anymore customers from quene $\pi_{n}^{q}$ in $\left[\pi_{n}^{\epsilon}, \infty\right)$. By convention. we shall assume in the secuel that $\pi_{m+1}^{\epsilon}=\infty$ whenever $m=\infty$.

From $\mathcal{I}$ we construct a new policy $\gamma$ as follows (cf. Figure 1):

- $\gamma$ follows $\pi$ in $\left[0, \pi_{n}^{e}\right)$ and $\gamma$ follows $\pi$ in $\left[\pi_{m+1}^{\prime}, \infty\right)$ if $m<\infty$, that is

$\star \gamma_{j}^{a}=\pi_{j}^{a}$ and $\gamma_{j}^{q}=\pi_{j}^{q}$ for $j=1, \ldots, n-1, \gamma_{j}^{\epsilon}=\pi_{j}^{\epsilon}$ for $j=1,2 \ldots \ldots n$;

$\star \gamma_{j}^{a}=\pi_{j}^{a}, \gamma_{j}^{\varphi}=\pi_{j}^{q}$ and $\gamma_{j}^{\epsilon}=\pi_{j}^{e}$ for $j \geq m+1$ if $m<\infty$;

- $\gamma_{n}^{a}=1, \gamma_{n}^{q}=\pi_{n}^{q}$. If $m<x$ then $\gamma_{n+1}^{\epsilon}-\gamma_{n}^{\prime}=\sigma_{s_{n}^{\pi}+1}^{\pi}$ i otherwise $\gamma_{n+1}^{f}-\gamma_{n}^{\prime}=\sigma_{v+1}^{\pi_{n}^{4}}$, where $v$ is the number of customers served at queue $\pi_{n}^{q}$ in $\left[0, \pi_{n}^{e}\right]$;

- For $j=n+1, \ldots, m, \gamma_{j}^{a}=\pi_{j-1}^{q}, \gamma_{j}^{q}=\pi_{j-1}^{q}$, and

$\star \gamma_{j+1}^{e}-\gamma_{j}^{\epsilon}=\pi_{j}^{e}-\pi_{j-1}^{e}$ if $\gamma_{j}^{e} \in\{0.2\}$

$\star \gamma_{j+1}^{e}-\gamma_{j}^{e}=\sigma_{s_{j-1}^{\pi}+1}^{\pi}$ if $\gamma_{j}^{a}=1$.

In other words, $\gamma$ behaves like $\pi$ except that during $\left[\pi_{n}^{e}, \pi_{m+1}^{e}\right) \gamma$ first serves a customer and then does what $\pi$ has been doing in $\left[\pi_{n}^{e}, \pi_{m}^{e}\right)$ if $m<\infty$ (resp. in $\left[\pi_{n}^{e}, \infty\right)$ if $m=\infty$ ). (Note that $\gamma_{m+1}^{e}=\pi_{m+1}^{e}$ if $m<\infty$.) 
Policy $\pi$ :

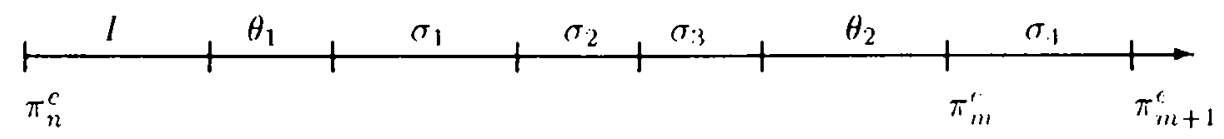

Policy $\gamma$ :

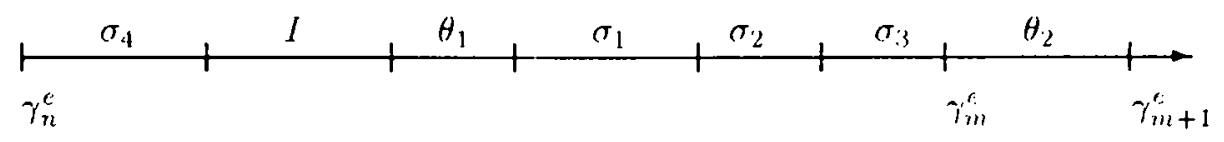

$I$ : Idle period: $\theta_{1}, \theta_{2}$ : Switching periods; $\sigma_{1}, \ldots, \sigma_{1}$ : Service periods.

Figure 1: An example of the behavior of $\pi$ and $\gamma$ in $\left[\pi_{n}^{c}, \pi_{m+1}^{e}\right)$.

Let us first show that $\gamma$ is admissible. This is true in $\left[0, \pi_{n}^{c}\right)$ since both policics $\pi$ and $\gamma$ are identical in this time interval. In $\left[\pi_{n}^{\epsilon}, \pi_{n+1}^{\epsilon}\right]$ this result is a consequence of the properties P1 and P2. Indeed, the above construction implies that for all $l=n . n+1 \ldots, m-1, i \in \mathbf{I}$,

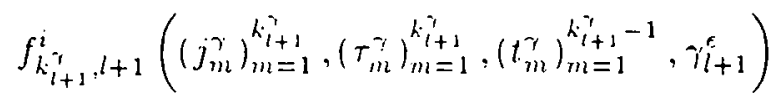

$$
\begin{aligned}
& \geq f_{k_{l+1}^{\gamma}, l}^{i}\left(\left(j_{m}^{\gamma}\right)_{m=1}^{k_{t+1}^{\gamma}},\left(\tau_{m}^{\gamma}\right)_{m=1}^{k_{i+1}^{\gamma}},\left(t_{m}^{\gamma}\right)_{m=1}^{k_{t+1}^{\gamma}-1}, \gamma_{++1}^{i}\right) \text {. } \\
& \geq f_{k_{i}, l}^{i}\left(\left(j_{m}^{\pi}\right)_{m=1}^{k_{i}^{\pi}},\left(\tau_{m i}^{\pi}\right)_{m=1}^{k_{i}^{\pi}},\left(t_{m}^{\pi}\right)_{m=1}^{k_{i}^{\pi}-1}, \pi_{l}^{e}\right),
\end{aligned}
$$

The inequality (3.2) follows from P2. The ineguality (3.3) follows from P1 together with the obvious relations $k_{l+1}^{\gamma}=k_{l}^{\pi}, \tau_{l}^{\gamma} \geq \tau_{l}^{\pi}, t_{l}^{\gamma} \geq l_{l}^{\pi}$ and $\gamma_{l+1}^{\epsilon} \geq \pi_{l}^{\epsilon}$ for all $l=n, n+1, \ldots, m-1$. We may therefore deduce from (2.4) and (3.3) that at time $\gamma_{l+1}^{c}$ the policy $\gamma$ has always as much information as $\pi$ at time $\pi_{l}^{e}$, for all $l=n, n+1, \ldots, m-l$. Further, at time $\gamma_{l+1}^{e}$, the policy $;$ is able to retrieve the history of policy $\pi$ at time $\pi_{l}^{e}$ (i.e., $H_{l}^{\pi}$ ) from its set of information, for all $l=n, n+1, \ldots m-1$. This explains why $y$ is admissible in $\left[\pi_{n}^{c}, \pi_{i n+1}^{c}\right)$. 
To show that the policy $\gamma$ is admissible in $\left[\pi_{n+1}^{\prime}, \infty\right)$, observe that $k_{i}=h_{i}, \gamma_{i}^{\prime}=\pi_{i}^{\prime}, r_{i}^{i}=\tau_{i}^{\pi}$ and $t_{l}^{\gamma}=t_{l}^{\pi}$ for all $l \geq m+1$. This yiclds, for all $l \geq m+1, i \in \mathbf{I}$, cf. P 1 .

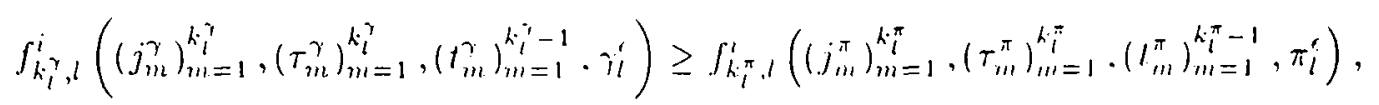

which shows that $\gamma$ always has as much information as $\pi$ in $\left[\pi_{m+1}^{c} \cdot \infty\right)$, which in turn shows that $\gamma$ may indecd follow $\pi$ in this time interval.

Let us now prove (3.1). It is easily seen from the construction of $y$ that this policy belongs to Il. Further,

$$
\begin{aligned}
& U^{\gamma}(t) \leq l^{\pi}(t), \text { for } t \in\left(\pi_{n}^{\prime}, \pi_{m+1}^{t}\right) \\
& U^{\gamma}(t)=l^{i \pi}(t) \text {. for } t \in\left[0, \pi_{n}^{c}\right] \cup\left[\pi_{m+1}^{\epsilon}, x\right) .
\end{aligned}
$$

Morcover, the policy $\gamma$ is greedy up to $\gamma_{n+1}^{c}$. Where $\gamma_{n+1}^{c}>\pi_{n}^{c}$. In the same manner, we can construct from $\gamma$ a policy $\gamma^{\prime} \in$ Il that is greedy n] to $\gamma_{n+1}^{\prime c}$, where $\gamma_{n+1}^{\prime \prime}>\gamma_{n+1}^{\prime \prime}$, and such that $U^{\gamma^{\prime}}(t) \leq U^{\pi}(t)$ for all $t \geq 0$.

Iterating this procedure we finally end up with a policy $\xi \in$ II that is greedy in $[0, \infty)$, and such that $U^{\xi}(t) \leq U^{\pi}(t)$ for all $t \geq 0$. The proof is then concluded by removing the conditioning on the input sequence $I$.

Proposition 3.2 Assume that A1 and A3 hold. Then. for any polisy $\pi \in$ II there cxists a policy $\xi \in \Gamma$ such that

$$
Q^{\xi}(t) \leq s t Q^{\pi}(t)
$$

for all $t \geq 0$.

Proof. Let us consider a realization $I$ of the input sequence $\left\{a_{n}, u_{n}, \sigma_{n}^{i}, \theta_{n}^{\prime \prime k},(i, j, k) \in \mathrm{I}^{3}, j \neq k\right\}_{n=1}^{\infty}$. Let $\pi$ be an arbitrary policy in $\pi \in I I-I^{\prime}$ (if $\pi \in \Gamma$ then take $\xi=\pi$ ) and lot $\pi$ run on the input sequence $I$.

On the sample path $\mathcal{I}$ generated by letting $\pi$ run on the input secuence. ler $n \geq 1$ be the smallest integer such that $\pi_{n}^{u}=0$ and $Q_{\pi_{n}^{q}}^{\pi}\left(\pi_{n}^{\epsilon}\right)>0$. Let $m$ be the smallest integer such that $n<m, \pi_{m}^{\prime \prime}=1$, and $\pi_{m}^{q}=\pi_{n}^{q}$. Note that if $m=\infty$ then the server does not server anymore customers from quene $\pi_{n}^{q}$ in $\left[\pi_{n}^{e}, \infty\right)$. By convention. we shall assume in the sequel that $\pi_{m+1}^{\prime}=\infty$ whenever $m=\infty$.

From $\mathcal{I}$ we construct a new policy a as follows (cf. Figure 2):

- $\gamma$ follows $\pi$ in $\left[0, \pi_{n}^{c}\right)$ and $\gamma$ follows $\pi$ in $\left[\pi_{m+1}^{\epsilon}, \infty\right)$ if $m<\infty$, that is

$$
\star \gamma_{j}^{a}=\pi_{j}^{a} \text { and } \gamma_{j}^{q}=\pi_{j}^{q} \text { for } j=1, \ldots n-1 . \gamma_{j}^{e}=\pi_{j}^{\epsilon} \text { for } j=1,2, \ldots n \text { : }
$$


$\star \gamma_{j}^{\prime \prime}=\pi_{j}^{\prime \prime}, \gamma_{j}^{\prime \prime}=\pi_{j}^{\prime \prime}$ and $\gamma_{j}^{\prime}=\pi_{j}^{\prime}$ for $j \geq m+1$ if $m<\infty$ :

- $\gamma_{n}^{a}=1 \cdot \gamma_{n}^{u}=\pi_{n}^{q}$ and $\gamma_{n+1}^{\prime}-\gamma_{n}^{\prime}=\sigma_{s_{n}^{T}+1}^{r}$;

- For $j=n+1 \ldots, m, \gamma_{j}^{n}=\pi_{j-1}^{\prime \prime}, \gamma_{j}^{\prime \prime}=\pi_{j-1}^{\prime \prime}$ and

$\star \gamma_{j+1}^{c}-\gamma_{j}^{c}=\pi_{j}^{c}-\pi_{i-1}^{c}$ if $\gamma_{j}^{\prime \prime} \in\{0.2\}$ :

$\star \gamma_{j+1}^{\epsilon}-\gamma_{i}^{r}=\sigma_{s j+1}^{\bar{j}}$ if $\gamma_{j}^{\prime \prime}=1$.

Policy $\pi$ :

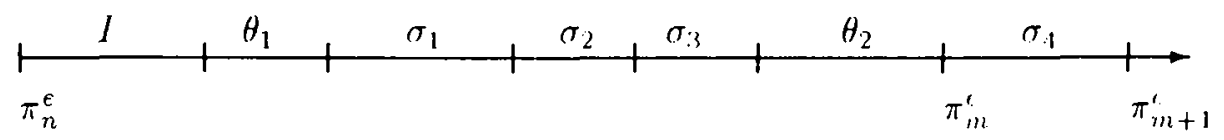

Policy $\uparrow$ :

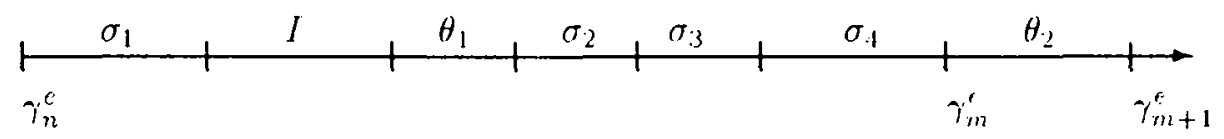

I: Idle period; $\theta_{1}, \theta_{2}$ : Switching periods: $\sigma_{1}, \ldots, \sigma_{4}$ : Service periods.

Figure 2: An example of the bchavior of $\pi$ and $\gamma$ in $\left[\pi_{n}^{c}, \pi_{m+1}^{r}\right)$.

The proof that $\gamma$ is admissible is identical to the proof given in Proposition 3.1.

With this construction, it is easily seen that $\gamma \in l l$ and that $\gamma_{m+1}^{e}=\pi_{m+1}^{\epsilon}$. Further,

$$
\begin{aligned}
& Q^{\gamma}(t) \leq Q^{\prime}(t), \text { for } t \in\left[\gamma_{n+1}^{c}, \pi_{m+1}^{\epsilon}\right) \\
& Q^{\gamma}(t)=Q^{-}(t), \text { for } t \in\left[0, \gamma_{n+1}^{e}\right) \cup\left[\pi_{n+1}^{\epsilon}, \infty\right) .
\end{aligned}
$$

Moreover, the policy $\gamma$ is greedy up to $\gamma_{n+1}^{\prime}$. where $\gamma_{n+1}^{\epsilon}>\pi_{n}^{e}$.

Iterating this procedure we fully end up with a policy $\xi \in \Gamma$ that is greedy in $[0, \infty)$, and such that $Q^{\gamma}(t) \leq Q^{\pi}(t)$ for all $t \geq 0$. The proof is then concluded by removing the conditioning on the input sequence $I$. 


\section{Optimality of exhaustive policies}

In view of the above results, it remains to compare the decisions between "serving" and "switching" in order to determine the optimal decision when the server is at a nonemply queue. We show that any policy in II can be improved by a greedy and exhanstive policy. In outher words, in order to stochastically minimize the unfinished work and the number of rustomers in the system at all time, the server can neither iclle at a nonemply enene nor leave the cusure before it is empty.

Proposition 4.1 Assumc that A1 holds. Then. for any policy $\pi \in$ Il there caists a policy $\xi \in I \cap \cap \Xi$ such that

$$
l^{\dot{\varepsilon}}(t) \leq_{s !}\left(l^{\pi}(t)\right.
$$

for all $t \geq 0$.

Proof. Owing to Proposition 3.1 we may restrict ourselves to the policies in l. The proof is identical to the proof of Proposition 3.1 except that $n$ is now defincel to be the first integer such that $\pi_{n}^{a}=2$ and $Q_{\pi_{n-1}^{a}}^{\pi}\left(\pi_{n}^{e}\right)>0$ (i.e., al time $\pi_{n}^{e}$ the decision is made to move the server although the queue where it is is located is not empty). The rest of the proof is analogons to that of the proof of Proposition 3.1, and it is therefore omitted.

Proposition 4.2 Assume that A1 and A3 hold. Then, for any policy $\pi$ E II there exists a policy $\xi \in \Gamma \cap \equiv$ such that

$$
Q^{\xi}(t) \leq_{s t} Q^{\pi}(t)
$$

for all $t \geq 0$.

Proof. The proof is identical to the proof of l'roposition 4.1 (simply replace "Proposition 3.1" by "Proposition 3.2"), and it is therefore omitted.

\section{Optimality of Stochastically Largest Queue Policies}

This section focuses on the choices of the queues to be visited when the server leaves a queue. Specifically, we define the class of Stochasticrlly Largest Queue (SLQ) policies, $\Sigma$, and prove that for any policy in $\Pi$ there exists a policy in $\Xi$ that will perform at least as 1 coll if the polling system is symmetric.

In general, it is not easy to iclentify the best policy within $\Sigma$. We will conclude this section with a number of interesting examples where it is possible to identify the optimal policy within $\Sigma$ and, thus, within II.

With any policy $\pi \in \Pi$, we associate the relations $\left\{R_{n}^{\pi}\right\}_{n=1}^{\infty}$, where $(j, k) \in R_{n}^{\pi}$ at the $n$-th decision epoch if one of the following two conditions holds: 
i) $F_{j, n}^{\top \pi}>F_{k, n}^{\pi}$ and $Q_{j}^{\pi}\left(l_{j, n}^{\pi}\right) \leq Q_{k}^{\pi}\left(l_{h, n}^{\pi}\right)$;

ii) $F_{j, n}^{\pi}=F_{k, n}^{\pi}$ and $Q_{, i}^{\pi}\left(F_{j, n}^{\pi}\right)<Q_{k}^{\pi}\left(l_{k, n}^{\prime \pi}\right)$

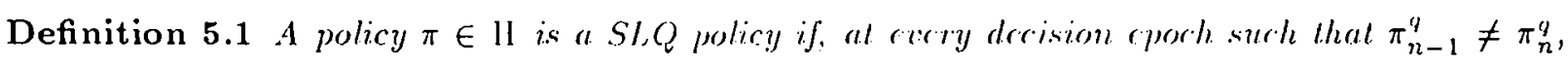
there caist.s no $k \in \mathbf{I}$ such that $\left(\pi_{n}^{q}, k\right) \in R_{n}^{;}$.

In the remainder of this paper, we shall assume that the polling system under consideration is symmetric, i.e., the assumptions A2-A5 are satisfied. As a consequener of A3, we may now assume without loss of generality that he serviec times are associated with the server. Let $\sigma_{n}$ be the $n$-th service time delivered by the server (observe that $\sigma_{n}$ docs not depend on we enforced polling policy). Likewise, because of assumption A4 we may also assume that the duration of the consecutive switching periods do not depend on the routing policy. Lat $\theta_{n}$ be the duration of the $n$-th switching period.

The main result in this section is the following.

Proposition 5.1 .1ssume A2. A3. A4 and A5 hold. Then, for any policy $\pi \in$ II, llerre axists a palicy $\xi \in \Sigma$ such that

$$
\begin{aligned}
& l^{\xi}(t) \quad \leq_{s t} \quad \dot{U}^{\pi}(t) \\
& Q^{\xi}(t) \leq_{s t} \quad Q^{\pi}(t)
\end{aligned}
$$

for all $t \geq 0$.

Before proving this assertion, we state the following lemma:

Lemma 5.1 Let $\pi \in \mathrm{Il}$ be an arbitrary policy. Fix $n \geq 1$ and $p, \eta \in\{1.2 \ldots . N\}$. From the sequences $\left\{a_{l}\right\}_{l=1}^{\infty},\left\{\sigma_{l}\right\}_{i=1}^{\infty}:\left\{\theta_{l}\right\}_{l=1}^{\infty}$ and $\left\{u_{l}\right\}_{l=1}^{\infty}$, we generale a new sequener $\left\{u_{l}^{\prime}\right\}_{l=1}^{\infty}$ as follows:

$$
\begin{aligned}
& u_{l}^{\prime}=u_{l}, \text { for alll such that } a_{l}<F_{p, n}^{r} ; \\
& u_{l}^{\prime}=u_{l} 1\left(u_{l} \neq p, u_{l} \neq q\right)+p 1\left(u_{l}=q\right)+q 1\left(u_{l}=p\right) \text {. for all l such that } a_{l} \geq F_{p, n}^{\pi} .
\end{aligned}
$$

Then, $\left\{u_{l}\right\}_{l=1}^{\infty}$ and $\left\{u_{l}^{\prime}\right\}_{i=1}^{\infty}$ are irlentical in law and $\left\{u_{l}^{\prime}\right\}_{l=1}^{\infty}$ is independent of $\left\{a_{l}, \sigma_{l}, \theta_{l}\right\}_{l=1}^{\infty}$.

The proof of Lemma 5.1 is given in Appendix A.

\section{Proof of Proposition 5.1.}

Let us consider a realization $I$ of the input secuence $\left\{a_{n}, u_{n}, \sigma_{n}, \theta_{n}\right\}_{n=1}^{\infty}$. As a result of Propositions 3.2 and 4.2 we can restrict ourselves to greedy and exhaustive policies. Let $\pi$ be an arbitrary policy 
in I’ $\cap \equiv \cap(I I-\Sigma)$ (if $\pi \in I^{\prime} \cap \Xi \cap \Sigma^{\prime}$ then talke $\xi=\pi$ ) such that the Sl, $($ rule is violated on the input sequence $I$.

On the sample path $\mathcal{I}$ generated by letting policy $\pi$ run on the inpul sexuence $I$, let $n \geq 1$ be the smallest integer such that $\pi_{n}^{a}=2$ and such that there exists some $h_{i} \in \mathrm{I}$ with $\left(\pi_{n}^{\prime}, h\right) \in R_{n}^{\pi}$ and where $\left(k, k^{\prime}\right) \notin R_{n}^{\pi}$ for all $k^{\prime} \in \mathrm{I}-\{h\}$.

Let $j:=\pi_{n}^{q}$ (i.e., the server switches to (quene $j$ in the interval of time $\left(\pi_{n}^{r}, \pi_{n+1}^{r}\right)$ ). Also defise

$$
i:=Q_{k}^{\pi}\left(F_{i, n}^{\pi}\right)-Q_{j,}^{\pi}\left(l_{j, n}^{\pi}\right)
$$

Observe that $K \geq 0$. This follows from the fact that $(j, k) \in R_{n}^{\pi}$ as well as from the fact that the mapping $x \rightarrow Q \bar{k}(x)$ is nondecreasing in $\left[f_{k, n}^{\pi}, f_{j, n}^{\prime \pi}\right]$ becausc of P3.

Let

$$
M_{i j}:=\inf \left\{l \in \mathbb{N} \mid l \geq n+1, Q_{,}^{\pi}\left(\pi_{l}^{i}\right)=0\right\} .
$$

In words, $\pi_{M}^{e}$, is the first time in $\left[\pi_{n+1}^{c}, \infty\right)$ when quene $j$ is empty (recall that the server arrives at queue $j$ at time $\pi_{n+1}^{e}$ ). If $Q_{j}^{\pi}(t)>0$ for all $t \geq \pi_{n+1}^{e}$, then we take $M_{j}=x$.

Let

$$
M^{k}:=\inf \left\{l \in \mathbb{N} \mid \pi_{M,+l}^{\prime}=k: \text { and } \pi_{M,+l}^{u}=1\right\}
$$

In words, $\pi_{M_{j}+\Lambda^{k}}^{e}$ is the first time when a customer of (quene $k$ is served in $\left[\pi_{M_{j}}^{e}, \infty\right)$ under policy $\pi$. In fact, as $\pi$ is greedy and exhaustive, $\pi_{N_{j}+N^{k}}^{e}$ is the first time when the server arrives at guene $k$ in $\left.\pi_{M j}^{e}, \infty\right)$. We assume that $M^{k}=\infty$ if queue $k$ is not visited by the server in $\left[\pi_{M_{j}}^{c}, x\right)$ under policy $\pi$.

From $\mathcal{I}$ we construct a new policy $y$ as follows (cf. Figure 3 ):

- For $1 \leq m \leq n-1, \gamma_{m}^{a}=\pi_{m}^{a}, \gamma_{m}^{q}=\pi_{m}^{q}, \gamma_{m+1}^{c}-\gamma_{m}^{\epsilon}=\pi_{m+1}^{c}-\pi_{m}^{c}$;

- $\gamma_{n}^{a}=\pi_{n}^{a}, \gamma_{n}^{q}=k, \gamma_{n+1}^{\epsilon}-\gamma_{n}^{\epsilon}=\pi_{n+1}^{\epsilon}-\pi_{n}^{\epsilon}$;

- For $n+1 \leq m \leq M_{j}+K-1, \gamma_{m}^{a}=1$. $\gamma_{m}^{\prime \prime}=h, \gamma_{m+1}^{\epsilon}-\gamma_{m}^{\epsilon}=\sigma_{s_{m+1}^{\dagger}}$;

- For $M_{j}+K \leq m \leq M_{j}+K+M^{k}-1$.

$\star \gamma_{m}^{a}=\pi_{m-K^{\circ}}^{a} ;$

$\star \gamma_{m}^{q}=1\left(\pi_{m-K}^{q} \notin\{j, k\}\right) \pi_{m-K}^{q}+1\left(\pi_{m-K}^{q}=j\right) k+1\left(\pi_{m-K}^{q}=k\right) j ;$

$\star \gamma_{m+1}^{e}-\gamma_{m}^{e}=\pi_{m+1-\kappa}^{e}-\pi_{m-K}^{\epsilon}$ if $\gamma_{m}^{a} \in\{0,2\}$;

$\star \gamma_{m+1}^{e}-\gamma_{m}^{e}=\sigma_{s_{m+1}^{\gamma}}$ if $\gamma_{m}^{\prime \prime}=1$;

- For $m \geq M_{j}+K^{*}+M^{k}$, 
$\star \gamma_{m}^{n}=\pi_{m}^{\prime \prime}$

$\star \gamma_{n}^{c}=\pi_{m}^{\prime}$;

$\star \gamma_{m}^{q}=1\left(\pi_{m}^{q} \notin\{j, h\}\right) \pi_{m}^{\prime \prime}+1\left(\pi_{m}^{\prime \prime}=j\right) l:+1\left(\pi_{m}^{\prime \prime}=l_{i}\right) j$.

Policy $\pi$ :

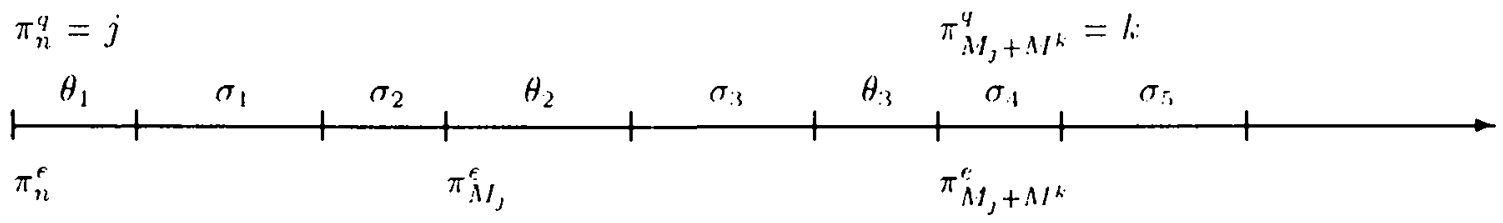

Policy $\gamma$ :

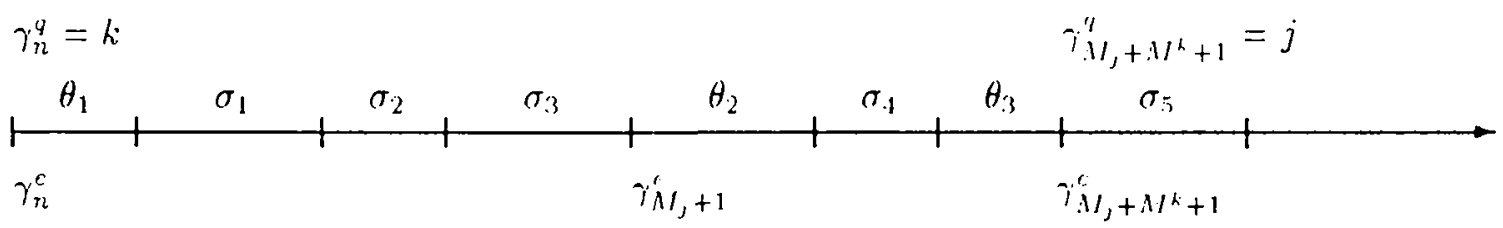

Figure 3: An example of the behavior of $\pi$ and $\gamma$ in $\left(\pi_{n}^{e}, \pi_{N_{j}+N^{k}+k+1}^{e}\right)$ willh $h=1$.

The policy $\gamma$ operates on the secuences $\left\{u_{1}\right\}_{i=1}^{\infty},\left\{u_{l}^{\prime}\right\}_{n=0}^{\infty},\left\{\sigma_{l}\right\}_{i=1}^{\infty}$ and $\left\{\theta_{l}\right\}_{i=1}^{\infty}$, where

$$
u_{l}^{\prime}= \begin{cases}u_{l}, & \text { if } u_{l}<F_{j, n}^{\pi} \text { or } u_{l} \notin\{j, k\} ; \\ k, & \text { if } u_{l} \geq F_{j, n}^{\pi} \text { and } u_{l}=j ; \\ j, & \text { if } u_{l} \geq F_{j, n}^{\pi} \text { and } u_{l}=k,\end{cases}
$$

for all $l \geq 1$. Owing to Lemma 5.1 , the secpuences $\left\{a_{l}\right\}_{l=1}^{\infty},\left\{u_{l}^{\prime}\right\}_{n=0}^{\infty},\left\{\sigma_{l}\right\}_{l=1}^{\infty}$ and $\left.\left\{\theta_{l}\right\}\right\}_{i=1}^{\infty}$ satisfy the assumptions A2-A5 and have the same joint distribution as $\left\{a_{l}\right\}_{l=1}^{\infty}:\left\{n_{l}\right\}_{n=0}^{\infty} \cdot\left\{\sigma_{l}\right\}_{l=1}^{\infty}$ and $\left\{\theta_{l}\right\}_{l=1}^{\infty}$.

We are going to show that the following relations are satisfied:

$$
\begin{aligned}
& \gamma_{M,+K+l}^{e} \geq \pi_{i j, l}^{e}, \text { for } l=0,1, \ldots, M^{k} \\
& \gamma_{M,+K+M^{k}}^{*}=\pi_{M,+K+M^{k}}^{*} \\
& Q_{\gamma_{i}^{\prime}(\gamma i)}^{\gamma(\gamma)}>0 \text {, if } \hat{i l}^{\prime \prime}=1, l \geq 1 \text {, }
\end{aligned}
$$


which will ensure that $\gamma$ is admissible. Indect. because of the structure of the information set (2.1), relation (5.s) ensures that, when $\gamma$ malies its $(l+1)$-st $(l \geq 0)$ decision in $[\gamma(M,+k, \infty)$ (which occurs at time $\gamma_{M,+K+l}^{r}$ ), it possesses at last as much information as $\pi$ when $\pi$ malkes the corresponding decision at time $\pi_{M_{j}+1}$. Property (5.10) cosures that $\gamma$ will never make the decision to serve an empty quenc.

Proof of $(5.8)$. Let $x_{l}$ be the total duration of the idling and switching periods in $\left[\pi_{M_{j}}^{e}, \pi_{M_{j}+l}^{e}\right)$. Let $L_{l}$ be the mumber of customers served by policy $\pi$ in the interval of time $\left[\pi_{A,}^{\prime}, \pi_{i, j}^{r}\right), 1 \geq 0$.

We have for $l=0,1, \ldots, M^{k}-1$,

$$
\begin{aligned}
\pi_{M_{3}+l}^{c} & =\pi_{M_{3}}^{\prime}+x_{l}+\sum_{i=1}^{L_{l}} \sigma_{s_{M_{3}}^{\pi}+i} \\
\gamma_{M_{j}+K+1}^{c} & =\gamma_{M_{3}}^{\prime}+x_{l}+\sum_{i=1}^{N+L_{l}} \sigma_{s_{M_{3}}^{\gamma}+i}
\end{aligned}
$$

Subtracting (5.11) from (5.12) yields

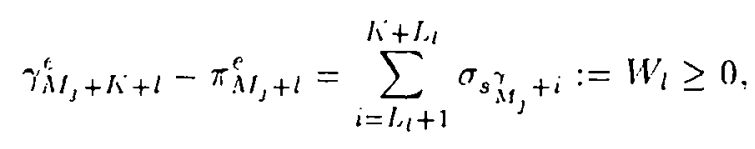

by noting that $\gamma_{M,}^{c}=\pi_{M}^{e}$, by construction of $\gamma$ and that $s_{M_{j}}^{\prime}=s \overline{M_{1}}$, from the definition (2.1).

Proof of (5.9). Since $Q_{k}^{\pi}\left(\pi_{M_{j}+M^{k}}^{e}\right) \geq K^{\circ}$ by definition of $M^{k}, K$ and $\pi$, we have

$$
\pi_{M_{j}+M^{k}+K}^{c}=\pi_{M_{j}+M^{k}}+\sum_{m=1}^{K} \sigma_{B_{M_{j}}+L_{M^{k}}+\cdots} .
$$

since $\pi$ is exhaustive and greedy, which in tum implies from (5.13) that

$$
\pi_{M_{1}+M^{k}+K^{\epsilon}}^{\epsilon}=\gamma_{M_{3}+M^{k}+K^{*}}^{e}
$$

which establishes $(5.9)$.

Proof of (5.10). First, it is clear that (5.10) holds for $l \leq M_{j}-1$ by definition of policy, . That (5.10) is true for $M_{j} \leq l \leq M_{j}+K-1$ comes from the fact that

$$
Q_{i}^{i}\left(\pi_{n}^{i}\right)=Q_{j}^{\pi}\left(\pi_{n}^{i}\right)+K
$$

which follows from (5.5) and from the definition of the sequence $\left\{u_{m}^{l}\right\}_{m=1}^{\sim}$. Therefore $Q_{\gamma_{l}^{q}}^{\gamma}\left(\gamma_{l}^{e}\right) \geq$ $I+M_{j}-l$ for all $M_{j} \leq l \leq M_{j}+K-1$. 
It remains to examine (5.10) for $l \geq M,+K$. led $l_{s}\left(l_{1}, l_{2}\right)$ denote the number of customers that arrive at queue $s \in \mathbf{I}$ in the time interval $\left(t_{1}, t_{2}\right]$ operating under $\pi .0<t_{1}<t_{2}$. Assume that $\gamma_{\Lambda,+k+r}^{a}=1$ for some $r \geq 0$. Dofine $i:=i l_{1,+k+,}$.

Then, it is easily seen by definition of $i$ l lath.

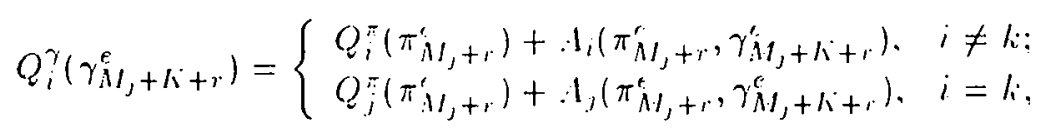

if $i \neq j$. Property $(5.10)$ then follows for $i \neq j$ becansse $Q_{i}^{\pi}\left(\pi_{M,+l}^{\prime}\right)>0$ (inderel, since $\pi$ is admissible and $\pi_{M,+l}^{\prime \prime}=1$ then necessarily $\left.Q_{i}^{\pi}\left(\pi_{M,+1}^{\prime}\right)>0\right)$.

We now examine the case where $i=j$. Observe that queue $j$ is nover sorved in $\left[\gamma_{n}^{\prime}, \gamma_{M_{3}+K+M^{k}}^{\prime}\right)$ under policy $\gamma$. Therefore, $(5.10)$ holds for $i_{l}^{\prime}=j$ and for all $l=1,2, \ldots, M l+M l^{k}+K-1$. The proof of $(5.10)$ is now concluded by noting that (5.16) and the definition of $\gamma$ y yeld

$$
Q_{i}^{\gamma}(t)=Q_{k}^{\pi}(t)
$$

for all $t \geq \gamma_{M,+K+\Lambda^{c}}^{c}$

We also deduce from (5.16) that for $t \geq \gamma_{N,+N+M k}$,

$$
\begin{aligned}
& Q_{i}^{\gamma}(t)=Q_{i}^{\pi}(t), \text { for } i \in \mathbf{I}-\{j, k\} ; \\
& Q_{j}^{\pi}(t)=Q_{k}^{i}(t) .
\end{aligned}
$$

that is both systems are again synchronized from time $\pi_{M I_{j}+M^{k}+K^{-}}^{+}$

In particular, relations $(5.17)-(5.19)$ indicate that $Q^{\gamma}(1) \leq Q^{\pi}(t)$ and $\ell^{\prime \gamma}(\ell) \leq l^{\prime \pi}(1)$ for $t \geq$ $\gamma_{M,+K+M^{k}}^{\epsilon}$

We now establish that $Q^{\gamma}(t) \leq Q^{\pi}(t)$ and $l^{\prime \gamma}(t) \leq U^{\pi}(t)$, for all $0 \leq t<i, i,+M^{k}+K^{*}$

Define $D^{\rho}(t)$ to be the number of departures in $[0 . t)$ under policy $\rho$. Recall that $s_{1}^{\prime \prime}$ is the number of departures in $\left[0, \rho_{l}^{c}\right)$ under $\rho$ (cf. $(2.1)$ ). It suflices to show that

$$
D^{\gamma}(t) \geq D^{\pi}(t) . \text { for all } t<\gamma_{M,+M^{k}+K}^{c}
$$

Proof of (5.20). The inequality (5.20) is clearly true for $0 \leq 1<$ iiis $+\kappa$ by defintion of $\gamma$ in $\left[0, \gamma_{M,+K}^{e}\right)$. Fix $\gamma_{M,+K}^{e} \leq t<\gamma_{M_{j}+M^{k}+K^{\prime}}^{\epsilon}$ and let $0 \leq 1 \leq M^{k}-1$ be such that

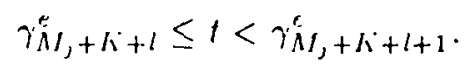




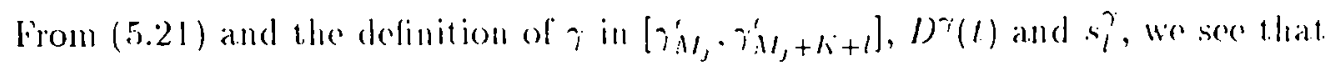

$$
\begin{aligned}
D^{\hat{\jmath}(1)} & =s_{M j+k+l}^{\gamma}, \\
& =s_{M_{j}+l}^{\pi}+k .
\end{aligned}
$$

On the other hand, (5.13) and $(5.21)$ yiodd $t<\pi_{1,+l+1}^{\prime}+W_{l+1}$.

Therefore,

$$
\begin{aligned}
D^{\pi}(1) & <s^{\pi}\left(\pi_{M_{1}+1+1}^{\prime}+W_{l+1}\right) \\
& \leq \cdot_{M_{1}+1+1}^{\pi}+k \\
& \leq 4_{M_{1}+1}^{\pi}+1+k
\end{aligned}
$$

and so $D^{\pi}(t) \leq s_{M,+1}^{\pi}+K=D^{\gamma}(1)$ from (5.22) (note that (5.23) and (5.24) follow from (5.13); (5.25) follows from the fact that at most one customer may be served between two consecutive decision epochs).

In summary, we have shown that $Q^{\top}(l) \leq Q^{\top}(l)$ and $U^{\top}(t) \leq U^{\top}(t)$ for all $l \geq 0$.

Although $\gamma$ may not be a greedy policy, arguments used in Propositions 3.1 and 3.2 can be used

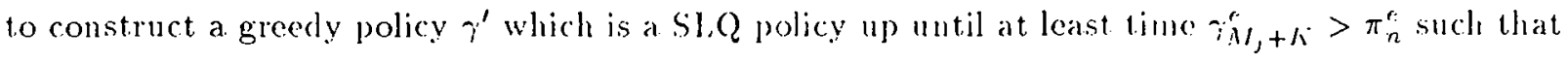
$U^{\gamma^{\prime}}(t) \leq U^{\gamma}(t) \leq U^{\pi}(t)$ and $Q^{\gamma^{\prime}}(t) \leq Q^{\gamma}(t) \leq Q^{-}(t)$, for all $t \geq 0$.

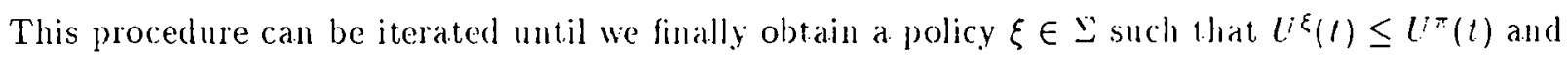
$Q^{\xi}(t) \leq Q^{\pi}(t)$, for all $t \geq 0$. The proof is then concluded by removing the conditioning on the input secuence.

In general, for a given set of functions $\left\{f_{i, 2}^{i}\right\}$ we are unable to jdentify the best sl,Q policy. This is due to the fact that the relation $R_{n}^{\pi}$ may not contain a single maximal element for all $n \geq 1$. However, in the first four information structures described in section 2 , we do identify the best SLQ policies:

1. Complete information. Here the SLQ policy is one that always switches the server to the queue with the largest queue size.

2. Partial information. Here the optimal SLQ policy is the cyclic poliry that begins with the first queue. Observe that this policy reguires no information except whether or not the quene currently being served is empty or not.

3. Periodic information. Here the optimal SLQ policy switches the server to the queue with the largest queue length at the time of the last update. If all of the queues have been visited since that update, then the server is switched to the queue visited the farthest in the past. 
4. Delayed information. The optimal Sl, Q policy belaves similaty to that for the system with periodic updates. The server is switched to the quene with the largest guene length that has not been visited since the last update and otherwise to the quene visited the farthest in the past.

Note that in the above four cases, the Sl, Q policies may not be unicue sine there may be several maximal elements in $R_{n}^{\pi}$ for some $n \geq 1$. However. sucle maximal dements $j$ and $f$ have the property that $F_{j, n}^{\pi}=H_{k, n}^{\pi}$ and that $Q_{j}^{\pi}\left(f_{j, n}^{\pi}\right)=Q_{k}^{\pi}\left(F_{j, n}^{\pi}\right)$. Therefore, all these SI $Q$ policies are optimal.

It is not possible to characterize the optimal SICQ policy for the system in which the controller obtains information reganding a cuene and it.s ncighbors (local informalion). However, the previous theorem does reduce the number of decisions that must be considered by the optimal policy.

\section{Optimality of Patient Policies}

We are now concerned with the optimal decision to be made when the server is at an empty queve. Should the server idle at the last visited quene or should it move to another quene? Consider first the case that the system is empty at the decision epoch. We will therefore assume that the controller has complete (i.e., instantaneous) information on the state of system, i.e., for all $\pi \in$ II, $n \geq 1$,

$$
r_{i, n}^{\pi}=\pi_{n}^{\epsilon}
$$

for all $i \in \mathbf{I}(\mathrm{cf} .(2.5)$.

For symmetric polling systems, we prove that optimal polling policies are within the class of patient policies $\Psi$, i.e., policies that decide to idle in the last visited gueuc when the system is empty.

Proposition 6.1 Assume A2, A3. A4 and A5 hold. Then, for any policy $\pi \in$ II. thenc exists a policy $\xi \in \mathrm{I} \cap \cap \equiv \cap \Psi$ such that.

$$
\begin{aligned}
& U^{\dot{\varepsilon}}(t) \leq s t \quad U^{\pi}(t) \\
& Q^{\dot{\varepsilon}(t)} \leq_{s t} Q^{\pi}(t),
\end{aligned}
$$

for all $t \geq 0$.

Proof. Consider a realization $I$ of the input sequence $\left\{a_{n}, u_{n}, \sigma_{n}, \theta_{n}\right\}_{n=1}^{\infty}$. As in the previons section, we restrict ourselves to greedy and exhanstive policies. Let $\pi$ be an arbitrary policy in $\Gamma \cap \equiv \cap(I I-\Psi)$ (if $\pi \in I^{\prime} \cap \equiv \cap \Psi$ then take $\xi=\pi$ ) such that $\pi$ is impatient on the input sequence $I$.

On the sample path $\mathcal{I}$ generated by letting policy $\pi$ run on the input sequence $I$, let $n \geq 1$ be the smallest integer such that $\pi_{n}^{a}=2$ and $Q_{i}^{\pi}\left(\pi_{n}^{e}\right)=0$ for all $i \in \mathrm{I}$. Let $j=\pi_{n}^{q}$ and $k=\pi_{n-1}^{q}$. From $\mathcal{I}$ we construct a new policy $\gamma$ as follows: 
- For $1 \leq m \leq n-1, \gamma_{m}^{\prime \prime}=\pi_{m}^{\prime \prime}, \gamma_{m}^{\prime}=\pi_{m}^{\prime \prime}, \gamma_{m}^{\prime}=\pi_{m}^{\prime}$;

- $\gamma_{n}^{\mathrm{a}}=0, \gamma_{n}^{q}=k \cdot \gamma_{n}^{\prime}=\pi_{n}^{r}$;

- For $m \geq n+1$;

$\star \gamma_{m}^{\prime \prime}=\pi_{m}^{\prime \prime}$;

$\star \gamma_{m}^{\prime}=\pi_{m}^{c}$

$\star \gamma_{m}^{\prime \prime}=1\left(\pi_{m}^{q} \notin\{j, k\}\right) \pi_{m}^{\prime \prime}+1\left(\pi_{m}^{\prime \prime}=j\right) k+1\left(\pi_{m}^{\prime \prime}=k\right) j$.

The policy $\gamma$ operates on the sequeners $\left\{a_{l}\right\}_{i=1}^{\infty},\left\{u_{l}^{\prime}\right\}_{n=1}^{\infty},\left\{\sigma_{l}\right\}_{l=1}^{\infty}$ and $\left\{\theta_{l}^{\prime}\right\}_{l=1}^{x}$. where for all $l \geq 1$,

$$
u_{l}^{\prime}= \begin{cases}u_{l}, & \text { if } a_{l} \leq \pi_{n}^{\epsilon} \text { or } u_{l} \notin\left\{j, k_{i}\right\} ; \\ l_{i} & \text { if } u_{l}>\pi_{n}^{\epsilon} \text { and } u_{l}=j ; \\ j, & \text { if } u_{l}>\pi_{n}^{\epsilon} \text { and } u_{l}=k,\end{cases}
$$

and

$$
\theta_{l}^{\prime}= \begin{cases}\theta_{l}, & \text { if } l<k_{n}^{\pi} ; \\ \theta_{l+1}, & \text { if } l \geq k_{n}^{\pi} .\end{cases}
$$

Recall that $k_{n}^{\pi}$ is the number of quenes visited by the server during $\left[0, \pi_{i}^{i}\right]$.

It can be shown (cf. Lemma 5.1) that the seduences $\left\{a_{l}\right\}_{i=1}^{\infty},\left\{u_{l}^{\prime}\right\}_{n=0}^{\infty},\left\{\sigma_{l}\right\}_{i=1}^{\infty}$ and $\left\{\theta_{l}^{\prime}\right\}_{l=1}^{\infty}$ satisfy the assumptions A2-A5 and have the same joint distribution as $\left\{a_{l}\right\}{\underset{l}{l=1}}_{1}^{\infty},\left\{u_{l}\right\}_{n=0}^{\infty},\left\{\sigma_{l}\right\}_{l=1}^{\infty}$ and $\left\{\theta_{l}\right\}_{l=1}^{\infty}$.

It is easy to sec from the definition of $\gamma$ and that of the secunences $\left.\left\{a_{l}\right\}_{i=1}^{\infty},\left\{u_{l}^{\prime}\right\}_{n=0}^{\infty},\left\{\sigma_{l}\right\}\right\}_{l=1}^{\infty}$ and $\left\{\theta_{l}^{\prime}\right\}_{l=1}^{\infty}$, that

$$
\begin{aligned}
& Q_{i}^{\gamma}(t)=Q_{i}^{\pi}(t), \quad \text { for all } 0 \leq t \leq \pi_{n}^{c}, i \in \mathrm{I} ; \\
& Q_{i}^{\gamma}(t)=Q_{i}^{\pi}(t), \quad \text { for all } t \geq 0 . i \in \mathrm{I}-\{j . h\} ; \\
& Q_{j}^{\gamma}(t)=Q_{i}^{\top}(t), \quad t>\pi_{n}^{\epsilon} ; \\
& Q_{k}^{\gamma}(t)=Q_{i}^{\top}(t) . \quad 1>\pi_{n}^{*},
\end{aligned}
$$

which imply that $\gamma$ is admissible and that $Q^{\gamma}(t)=Q^{\pi}(t)$ and $l^{\gamma}(t)=t^{\top}(t)$ for all $t \geq 0$.

Note that $\gamma$ may not be greedy as customers may arrive at queue $h$ during $\left(\pi_{n}^{\epsilon}, \pi_{n+1}^{\epsilon}\right)$. [ising the arguments of Propositions 3.2 and 4.2 yields a polling policy $\gamma^{\prime}$ which is greedy, exhaustive and patient until $\pi_{n+1}^{e}$ and such that

$$
\begin{array}{ll}
Q^{\gamma^{\prime}}(t) \leq Q^{\gamma}(t)=Q^{\pi}(t), & t \geq 0 ; \\
U^{\gamma^{\prime}}(t) \leq U^{\prime}(t)=U^{\pi}(t), & t \geq 0 .
\end{array}
$$


This procedure can be iterated until we finally obtain a policy $\xi \in \Psi$ such that $\|^{\xi}(1) \leq l^{\pi}(1)$ and $Q^{\xi}(t) \leq Q^{\top}(l)$, for all $t \geq 0$. The proof is then concluded by removing the conditioning on the input sequence $l$.

\section{$7 \quad$ Optimality of Non-Idling Policies}

We now consider the optimal decision to he male when the server is at, an emply quene while the system is not necessarily empty. Recall that a polling policy is an idling policy if the server stays idling at that empty quene. It is non-idling if the server switches to another queue (even if the latter is empty).

In general, non-idling policies are not optimal. llowever, when the polling system is symmetric and slotted, we show below that optimal policies are within the class of non-idling policies $\Upsilon$.

Proposition 7.1 Assume A2, A3. A4 and A5 hold. If $\theta_{n}=1$ a.s.. and $\sigma_{u}, \tau_{n} \in \mathbb{I N}_{+}$. lhen, for any policy $\pi \in I$, there caists a policy $\xi \in l^{\prime} \cap \Xi \cap \Upsilon$ such that.

$$
\begin{aligned}
& l^{\dot{\varepsilon}}(1) \quad \leq_{s l} \quad U^{\pi}(t) ; \\
& Q^{\dot{\varepsilon}(1)} \leq_{s t} \quad Q^{r}(1),
\end{aligned}
$$

for all $t \geq 0$.

\section{Proof.}

The proof is analogous to the proof of Proposition 5.1.

Consider a realization $I$ of the input secpuence $\left\{a_{n}, u_{n}, \sigma_{n}, \theta_{n}\right\}_{n=1}^{\infty}$. Owing to Propositions 3.2 and 4.2, we can restrict ourselves to greedy and cxlaustive policies. Let $\pi$ be an arbitrary policy in $\Gamma \cap \Xi \cap(I I-\Upsilon)$ (if $\pi \in \Gamma \cap \Xi \cap \Upsilon$ then take $\xi=\pi$ ) such that $\pi$ is idling on the input sequence $I$.

Under the assumptions of the proposition. we can assume without loss of generality that the durations of idling periods are all egual to one. Therefore, if for some $n \geq 1, \pi_{n}^{\prime \prime}=0$, then $\pi_{n+1}^{\epsilon}=\pi_{n}^{e}+1$.

On the sample path $\mathcal{I}$ generated by letting policy $\pi$ run on the injut secuence $I$, let $n \geq 1$ be the smallest in teger such that $\pi_{n}^{n}=0$ and $Q_{\sigma_{n}^{\prime \prime}}^{r}\left(\pi_{n}^{\prime}\right)=0$. Let $j=\pi_{n}^{\prime \prime}$ and $k \in \mathbf{I}-\{j\}$. Denote $K:=Q_{k}^{\pi}\left(\pi_{n}^{c}\right) \geq 0=Q_{j}^{\pi}\left(\pi_{n}^{\epsilon}\right)$.

As in the proof of Proposition 5.1, we define

$$
\begin{aligned}
& M_{j}=\inf \left\{l \in \mathbb{N} \mid l \geq n+1, Q_{j}^{\pi}\left(\pi_{l}^{e}\right)=0\right\} \\
& M^{k}=\inf \left\{l \in \mathbb{N} \mid \pi_{M_{j}+l}^{a}=k \text { and } \pi_{\Lambda_{3}+l}^{a}=1\right\}
\end{aligned}
$$


In words, $\pi_{n,}^{\prime}$ is the first time in $\left[\pi_{n+1}^{\prime}, x\right)$ when cuene $j$ is empty. Noto that there may be an arrival at quene $j$ at time $\pi_{n+1}^{r}$, in which case the sorver has to serve quene $j$ until the quene is empty, as $\pi$ is gredy and exhanstive. If $Q_{j} \pi(1)>0$ for all $l \geq \pi_{n+1}^{\prime}$, then we take $M_{l}=\infty$. The

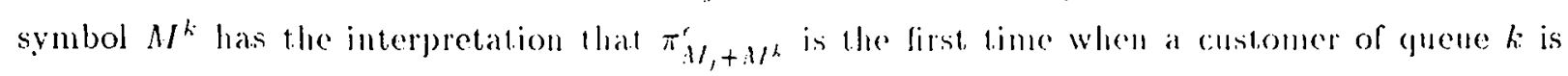
served in $\left[\pi_{M_{j}}^{k}, \infty\right)$ under policy $\pi$. Wo assume that $M^{k}=\infty$ if fuene k is not visited by the server in $\left[\pi_{i}^{c}, \infty\right)$ under policy $\pi$.

From $\mathcal{I}$ we construct a new policy if an follows:

- For $1 \leq m \leq n-1, \gamma_{m}^{a}=\pi_{m}^{\prime}, \gamma_{m}^{\prime \prime}=\pi_{m}^{\prime \prime} \cdot \gamma_{m+1}^{\prime}-\gamma_{m}^{\prime}=\pi_{m+1}^{e}-\pi_{m}^{\prime}$;

- $\gamma_{n}^{a}=2, \gamma_{n}^{q}=k, \gamma_{n+1}^{e}-\gamma_{n}^{c}=\pi_{n+1}^{c}-\pi_{i i}^{\prime}=1$ :

- For $n+1 \leq m \leq M_{i}+h-1 \cdot \gamma_{m}^{\prime \prime}=1 \cdot \gamma_{m}^{q}=k_{i} \gamma_{m+1}^{\prime}-\gamma_{m i}^{c}=\sigma_{s_{m+1}^{\prime}}$ :

- $\operatorname{lor} M_{j}+K \leq m \leq M_{j}+K+M^{k}-1$.

$\star \gamma_{m 2}^{\prime \prime}=\pi_{m-\Lambda}^{\prime \prime} ;$

$\star \gamma_{m}^{q}=1\left(\pi_{m-K}^{q} \notin\{j, l\}\right) \pi_{m-K}^{\prime \prime}+1\left(\pi_{m-K}^{q}=j\right) k+1\left(\pi_{m-\kappa}^{q}=k\right) j ;$

$\star \gamma_{m+1}^{e}-\gamma_{m}^{c}=\pi_{m+1-K}^{e}-\pi_{m-K}^{c}=1$ if $\gamma_{m}^{\prime \prime} \in\{0,2\}$;

$\star \gamma_{m+1}^{e}-\gamma_{m}^{e}=\sigma_{s_{m+1}^{3}}$ if $\gamma_{m}^{a}=1$ :

- For $m \geq M_{j}+K+M^{k}$,

$\star \gamma_{m}^{a}=\pi_{m}^{\prime \prime}$;

$\star \gamma_{m}^{e}=\pi_{m}^{e}$;

$\star \gamma_{m}^{q}=1\left(\pi_{m}^{q} \notin\{j, k\}\right) \pi_{m}^{q}+1\left(\pi_{m}^{\prime}=j\right) k+1\left(\pi_{m}^{q}=k\right) j$.

The policy $\gamma$ operates on the sequences $\left\{u_{l}\right\}_{i=1}^{\infty},\left\{u_{l}^{\prime}\right\}_{n=0}^{\infty},\left\{\sigma_{l}\right\}_{i=1}^{\infty}$ and $\left\{\theta_{l}\right\}_{l=1}^{\infty}$, where

$$
u_{l}^{\prime}= \begin{cases}u_{l}, & \text { if } u_{l} \leq \pi_{n}^{e} \text { or } u_{l} \notin\{j, k\} ; \\ k, & \text { if } u_{l}>\pi_{n}^{e} \text { and } u_{l}=j ; \\ j, & \text { if } u_{l}>\pi_{n}^{e} \text { and } u_{l}=k,\end{cases}
$$

for all $l \geq 1$. Similar to Lemma 5.1 , one can show that the sequences $\left\{a_{l}\right\}_{l=1}^{\infty} \cdot\left\{u_{l}^{\prime}\right\}_{n=0}^{x_{i}},\left\{\sigma_{l}\right\}_{l=1}^{\infty}$ and $\left\{\theta_{l}\right\}_{l=1}^{\infty}$ satisfy the assumptions A2-A5 and have the same joint distribulion as $\left.\{a l\}\right\}_{i=1}^{\infty},\left\{u_{l}\right\}_{n=0}^{\infty}$. $\left\{\sigma_{l}\right\}_{l=1}^{\infty}$ and $\left\{\theta_{l}\right\}_{i=1}^{\infty}$.

It is now immediate (by mimicling the proof of Proposition 5.1) that the policy $\gamma$ is admissible, and that

$$
Q^{\gamma}(t) \leq Q^{\pi}(t), \quad l^{\top}(t) \leq U^{\pi}(t), \quad t \geq 0
$$


Using again the same argument as these in the preof of propesstion 5.l. we obtain a policy $\xi \in \Upsilon$ such that $l^{\varepsilon}(1) \leq l^{\pi \pi}(1)$ and $Q^{\varepsilon}(1) \leq Q^{\pi}(1)$. for all $l \geq 0$.

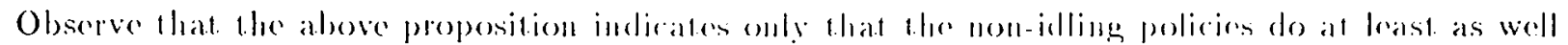

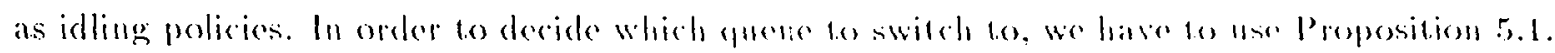

Combining the results of Propositions f.l and $i$.l we oltain that patient and impatient policies are both optimal in sloted systems:

Corollary 7.1 Assume: A2, A3. A4 and A5 hold. If $\theta_{n}=1$ a.s., and $\sigma_{n}, \tau_{n} \in \mathbb{I}-\{0\}$, thr:n, for

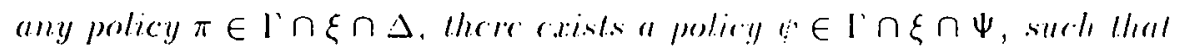

$$
l^{\pi}(t)={ }_{s t}(t), \quad Q^{\pi}(1)={ }_{s t} Q^{i \prime}(1), \quad \forall 1 \geq 0 .
$$

\section{A Appendix}

\section{Proof of Lemma 5.1.}

Let us first show that the secuences $\{m\}_{i=1}^{\infty}$ and $\left\{u_{l}^{\prime}\right\}_{i=1}^{\infty}$ are identicat in hall. Consider the case where $x_{1}=p, x_{2} \neq p, x_{2} \neq q, n_{1}<n_{2}$. Wo halle. (r. (5.3). (5.1),

$$
\begin{aligned}
& \mathrm{P}\left(u_{n_{1}}^{\prime}=x_{1}, u_{n_{2}}^{\prime}=x_{2}\right)
\end{aligned}
$$

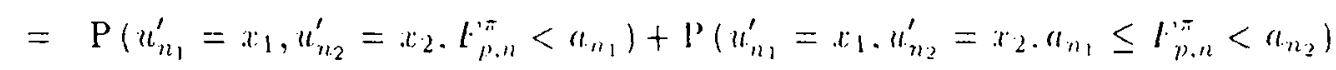

$$
\begin{aligned}
& +\mathrm{P}\left(u_{n_{1}}^{\prime}=x_{1}, u_{n_{2}}^{\prime}=x_{2}, u_{n_{2}} \leq P_{r_{1, n}}^{n_{1}}\right) \text {, }
\end{aligned}
$$

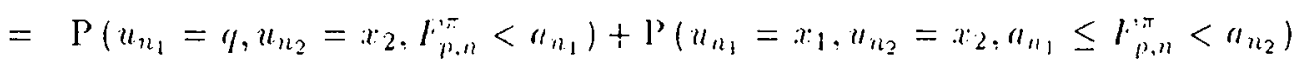

$$
\begin{aligned}
& +\mathrm{P}\left(u_{n_{1}}=x_{1}, u_{n_{2}}=x_{2}, u_{n_{2}} \leq P_{p, n}^{\pi}\right) \text {, } \\
& =\mathrm{P}\left(u_{n_{1}}=x_{1}, u_{n_{2}}=x_{2}\right) \text {. }
\end{aligned}
$$

where we have used the independence assumption between $\left\{u_{l}\right\}_{i=1}^{x}$ and $\left\{a_{l}, \sigma_{l} \text {. } \theta_{l}\right\}_{i=1}^{x}$. together with A5. The general proof is similar and is omitted for sake of concisencess.

It remains to establish that $\left\{u_{l}^{\prime}\right\}_{l=1}^{\infty}$ is independent of $\left\{u_{l}, \sigma_{l}, \theta_{l}\right\}_{i=1}^{\infty}$. Len us consider a realization $I$ of the input sequence $\left\{a_{l}, \sigma_{l}, \theta_{l}\right\}_{i=1}^{\infty}$.

For $x_{1}=p, x_{2} \neq p, x_{2} \neq q, u_{1}<u_{2}$. it is readily seen that, of. (5.3), (5.4).

$$
\begin{aligned}
& \mathrm{P}\left(u_{n_{1}}^{\prime}=x_{1}, u_{n_{2}}^{\prime}=x_{2} \mid I\right) \\
& =\mathrm{P}\left(u_{n_{1}}=q_{1}, u_{n_{2}}=x_{2} \cdot F_{n_{1}, n_{1}}^{\pi}<n_{n_{1}} \mid I\right)+\mathrm{P}\left(n_{n_{1}}=x_{1} \cdot u_{n_{2}}=x_{2} \cdot F_{P, n}^{n_{1}} \geq n_{n_{1}} \mid I\right) . \\
& =\mathrm{P}\left(u_{n_{1}}=q_{1} u_{n_{2}}=x_{2} \cdot \mid F_{p, n}^{\pi}<a_{n_{1}} . l\right) \mathrm{P}\left(H_{n, n_{1}}^{\pi}<a_{n_{1}} \mid l\right) \\
& +\mathrm{P}\left(u_{n_{1}}=x_{1}, u_{n_{2}}=x_{2} \mid I_{n, n}^{\pi} \geq a_{n_{1}}, I\right) \mathrm{P}\left(I_{n_{1}, n_{1}}^{\pi} \geq a_{n_{1}} \mid l\right) \text {. } \\
& =\mathrm{P}\left(u_{n_{1}}=x_{1}, u_{n_{2}}=x_{2}\right) \text {. }
\end{aligned}
$$




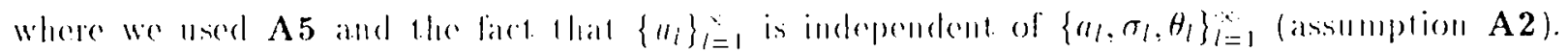
Next. using the property that $\{u\}_{i=1}$ and $\left\{u_{l}^{\prime}\right\}_{i=1}$ ane identical in law, we gen from (A.1).

$$
P\left(u_{n_{1}}^{\prime}=x_{1} \cdot u_{n_{2}}^{\prime}=x_{2} \mid l\right)=P\left(u_{n_{1}}^{\prime}=x_{1} \cdot u_{n_{2}}^{\prime}=x_{2}\right) .
$$

Again, the general proof is omitled for satie of conciseness.

\section{References}

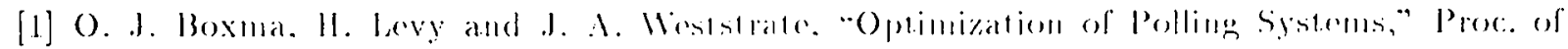

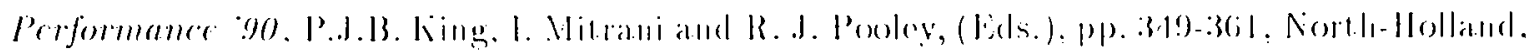
1990 .

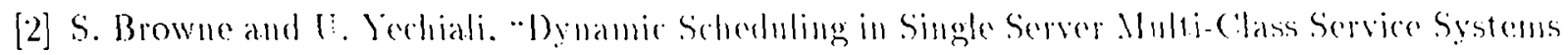

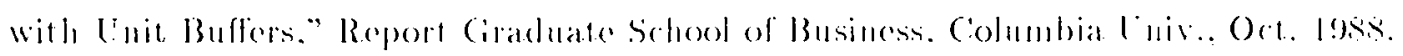

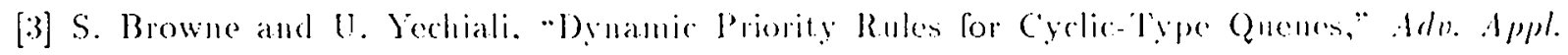
Prob. 21. pp. 432-150. 1939.

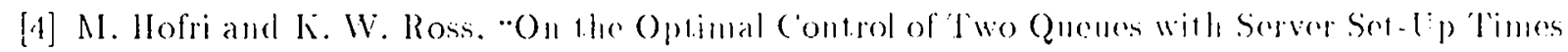

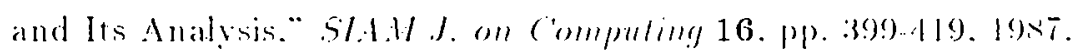

[5] II. Levy, M. Sidi. and (). J. Boxma. "I)ominance Revations in Polling Systems." Queuring Siystems (QTil:STA) 6. pp. 15.5-172. 1990.

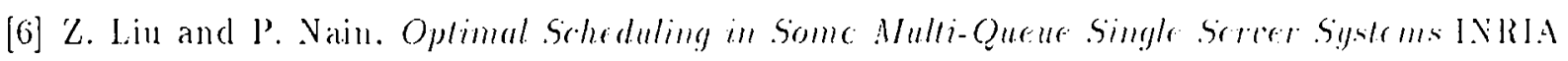

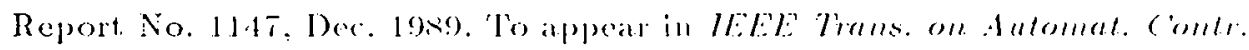

[7] Il. Takagi, Analysis of Polling Systcms. Mrl'T Press. 1986.

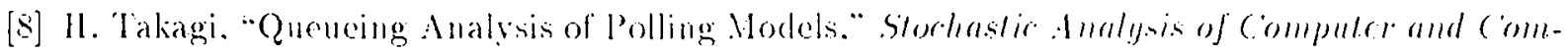
muniration Sy.stems. Il. Takiagi (Eld.). North Ilolland. 1990).

[9j D. Towsley, S. Folida. II. Santoso. "I)esign and Analysis of Flow Control Protocols for

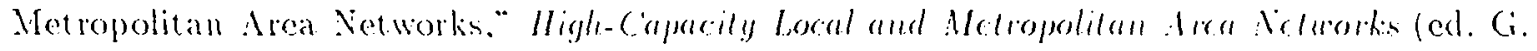
Pujolle), Springer Verlag. pp. 171-192. 1991. 
ISSN $0249-6399$ 